Mens

revue d'histoire intellectuelle de l'Amérique française

Olivier Marcil. La raison et l'équilibre. Libéralisme, nationalisme et catholicisme dans la pensée de Claude Ryan au Devoir (1962-1978). Montréal, Éditions Varia, 2002. 292 p. (Coll. « Histoire et Société »).

\title{
Michel Bock
}

Volume 4, numéro 1, automne 2003

URI : https://id.erudit.org/iderudit/1024636ar

DOI : https://doi.org/10.7202/1024636ar

Aller au sommaire du numéro

Éditeur(s)

Centre de recherche en civilisation canadienne-française

ISSN

1492-8647 (imprimé)

1927-9299 (numérique)

Découvrir la revue

Citer ce compte rendu

Bock, M. (2003). Compte rendu de [Olivier Marcil. La raison et l'équilibre. Libéralisme, nationalisme et catholicisme dans la pensée de Claude Ryan au Devoir (1962-1978). Montréal, Éditions Varia, 2002. 292 p. (Coll. « Histoire et Société »).] Mens, 4(1), 136-144. https://doi.org/10.7202/1024636ar d'utilisation que vous pouvez consulter en ligne. 
phe. Du point de vue du contenu également. Trop scolaire pour le grand public, trop mauvais pour des lecteurs avertis, l'ouvrage de Catherine Bouchard trouvera difficilement un public. On s'explique d'ailleurs mal qu'il ait pu trouver un éditeur.

\title{
Pascale Ryan \\ Département des lettres et communications \\ Université de Sherbrooke
}

\begin{abstract}
Olivier Marcil. La raison et l'équilibre. Libéralisme, nationalisme et catholicisme dans la pensée de Claude Ryan au Devoir (1962-1978). Montréal, Éditions Varia, 2002. 292 p. (Coll. "Histoire et Société ").
\end{abstract}

Les rayons de nos bibliothèques ne croulent pas sous le poids des ouvrages consacrés à l'œuvre et à la pensée de Claude Ryan. D'aucuns imputeront peut-être le phénomène à ce que l'ancien directeur du Devoir projetait une image plutôt terne de lui-même ou, à tout le moins, moins flamboyante que celle de ses deux grands adversaires politiques, Pierre Trudeau et René Lévesque. Les mauvaises langues, pour leur part, préféreront une explication d'ordre idéologique en affirmant que la voie médiane que Ryan a toujours tenté de tracer entre le fédéralisme centralisateur de l'un et l'option souverainiste de l'autre résultait de la tiédeur de ses propres convictions politiques. Souvent éclipsé par l'affrontement Trudeau - Lévesque, Claude Ryan n'en mérite pas moins de figurer parmi les intellectuels et hommes d'action les plus influents des dernières décennies. Olivier Marcil apporte une contribution précieuse à l'histoire des idées au Québec et au Canada en proposant une analyse fine et intelligente de l'idéologie de celui 
qui en viendrait à incarner la "troisième voie constitutionnelle » sur l'échiquier politique québécois, celle du fédéralisme «asymétrique ».

L'auteur tente de démontrer, en quatre chapitres bien construits, que Claude Ryan était non pas l'homme du compromis, mais bien celui de la pondération et de la modération, qu'il réussit à effectuer une synthèse cohérente et originale de trois grands mouvements d'idées parfois antinomiques en apparence - libéralisme, nationalisme et catholicisme - et que son apport au débat politique fut, somme toute, immense. Les trois premiers chapitres sont consacrés à l'équilibre parfois fragile que Ryan tenta d'établir et de garder entre ses valeurs libérales et nationalistes. Après son entrée au Devoir au début de la Révolution tranquille, il s'éloigna progressivement de la perspective universaliste qu'il avait adoptée lors de son passage à l'Action catholique de 1945 à 1962, l'analyse de l'actualité canadienne et québécoise au quotidien offrant une terre fertile à son nationalisme croissant. L'auteur identifie trois « moments-charnières » qui représentent autant d'étapes dans l'évolution de la pensée de Ryan. En 1965, la Commission royale d'enquête sur le bilinguisme et le biculturalisme déposa son rapport préliminaire, en même temps que le mouvement indépendantiste connaissait un essor remarquable. Ryan prit alors conscience du rapport inégal et parfois injuste qui unissait Canadiens français et anglais. Sans jamais abandonner sa foi en la primauté des droits individuels fondamentaux, il se refusa également à écarter du revers de la main les droits collectifs, contrairement à Pierre Trudeau qui fit son entrée sur la scène politique fédérale au même moment. En 1970-1971, d'ailleurs, le comportement de Trudeau lors de la Crise d'octobre et de la conférence constitutionnelle de Victoria lui révéla toute la rigidité du premier ministre et son incapacité à penser le Canada à l'extérieur du cadre de sa logi- 
que individualiste «atomiste ». Si le sentiment nationaliste de Ryan atteignit, durant cette période, son point culminant, il devait pourtant être tempéré par l'accession au pouvoir du Parti québécois en 1976 et, en particulier, par l'adoption de la Charte de la langue française qui subordonnait, à ses yeux, les droits individuels aux droits collectifs. Critique souvent outré des politiques linguistiques péquistes, il quitterait Le Devoir peu de temps après pour faire le grand saut en politique provinciale et assumer la direction du Parti libéral du Québec.

Marcil démontre de manière convaincante qu'il serait faux de voir en Claude Ryan un adversaire du nationalisme. Dans son édifice conceptuel, affirme l'auteur, les valeurs libérales et nationalistes se côtoyaient harmonieusement, bien que les premières dussent l'emporter sur les deuxièmes en cas de conflit. Le nationalisme de Ryan s'inscrivait résolument dans le cadre fédéral et s'inspirait largement de la thèse de la dualité canadienne, certes, mais le directeur du Devoir refusait d'emboîter le pas à Trudeau qui réduisait cette dualité à un phénomène purement et simplement linguistique. Au contraire, l'existence de deux communautés linguistiques au pays était selon lui l'expression de la cohabitation de deux communautés culturelles, le Canada français et le Canada anglais. Ryan déplora longuement que le bilinguisme officiel du gouvernement Trudeau eût été coupé de son cadre justificatif pourtant indissociable, le biculturalisme. Ainsi, les politiques linguistiques du gouvernement fédéral lui paraissaient impuissantes à mettre un terme à la crise que traversait le Canada depuis le début de la Révolution tranquille. Il se rallia peu à peu à l'idée qu'il incombait dorénavant au Québec de déterminer sa propre politique linguistique et de devenir, ce faisant, l'« expression politique » du Canada français.

Cela ne signifiait pas pour autant que le Québec et le Canada français se confondaient l'un avec l'autre. Voilà justement l'accusation qu'il portait contre Lévesque et les 
péquistes : ceux-ci, en mettant l'État québécois au service d'abord et avant tout de la majorité canadienne-française, sans égard aux autres groupes culturels qui s'y trouvaient, faisaient œuvre antilibérale, d'après lui. Ryan en viendrait à la conviction que le Québec était plutôt une "société » ou une "nation » (termes à peu près interchangeables dans son vocabulaire, nous explique Marcil) qui possédait une culture publique commune et qui se devait d'intégrer tous les individus en faisant partie. La distinction qu'il établissait entre les notions d'« intégration » et d'" assimilation » n'était pas fortuite, car les desseins légitimes de l'État, insistait-il, se limitaient à exiger que les minorités ethnoculturelles pussent remplir leurs devoirs de citoyens, sans plus. C'était ce lien citoyen, plutôt que l'origine ethnoculturelle, qui cimentait la «nation » québécoise et qui consacrait la définition libérale du nationalisme de Ryan. L'auteur précise d'ailleurs qu'après 1967, l'année des premières assises des États généraux du Canada français, le journaliste délaisserait progressivement les vocables « race / ethnie canadienne-française ", auxquels il préférerait de plus en plus les termes "société / nation / peuple québécois(e)».

Si le Québec était l'« expression politique » du Canada français, il était nécessaire pour que la dualité canadienne fût respectée de lui accorder une marge de manœuvre suffisamment grande pour qu'il pût assumer pleinement cette responsabilité. Le régime fédéral qu'envisageait Ryan aurait été largement décentralisé, quitte à ce que le Québec obtînt un statut particulier parmi les autres provinces. Le conflit qui l'opposa au premier ministre Trudeau était donc irréductible. Les politiques centralisatrices du gouvernement fédéral constituaient la négation de la dualité culturelle du Canada telle qu'il la concevait et du rôle de l'État québécois comme gardien de la composante minoritaire de cette dualité. La souverainetéassociation de René Lévesque, qu'il jugeait impraticable et irréaliste, ne le séduisait pas davantage. Il eût été impossible 
pour le Québec, estimait-il, de négocier d'égal à égal la mise en place d'un régime de type confédéral avec le reste du Canada: celui-ci possédait des intérêts trop divisés sur le plan régional pour qu'il pût bénéficier d'une unité d'action semblable à celle qui caractérisait le Québec. Ryan n'en démordrait pas : la décentralisation et l'obtention d'un statut particulier représentaient les meilleurs moyens pour le Québec d'assurer son développement socioculturel, politique et économique dans le respect des droits individuels que toute société démocratique et libérale se devait de protéger et de promouvoir.

Bien entendu, Ryan éprouverait le plus de difficulté à concilier ses principes libéraux et nationalistes lorsque se poserait la délicate question de la langue d'enseignement. Farouche opposant à la loi 101, il refusait de réduire les AngloQuébécois à une minorité " comme les autres » en raison du rôle historique qu'ils avaient joué au Québec depuis deux siècles. En revanche, il se montra prêt à accepter certaines mesures coercitives modérées en matière d'éducation afin de préserver le français comme langue commune dans l'espace public québécois. Renonçait-il de la sorte à ses convictions libérales? Son nationalisme l'emportait-il finalement sur son libéralisme? Non, explique Marcil, car selon Ryan, il était permis de prendre des mesures exceptionnelles s'inspirant d'un esprit de justice : la puissante minorité anglo-québécoise exerçait un pouvoir d'attraction sans commune mesure avec sa position démographique au Québec et qu'il fallait ramener à des proportions plus raisonnables.

Le quatrième et dernier chapitre aborde le rapport entre catholicisme et libéralisme dans la pensée de Claude Ryan, thème qui reçoit toutefois un traitement beaucoup plus restreint que celui qu'accorde Marcil à son nationalisme et à son libéralisme. Cette asymétrie, nous explique-t-il, est due au nombre nettement moins élevé de textes que consacra Ryan à 
la problématique religieuse durant son séjour au Devoir. Néanmoins, il eût été difficile de la passer complètement sous silence, compte tenu des convictions religieuses que Ryan n'a jamais cessé de professer, de son engagement antérieur à l'Action catholique et des bouleversements que traversa l'Église durant les années 1960 et 1970 au Québec comme ailleurs.

Encore une fois, Marcil soutient la thèse du caractère modéré de l'idéologie de Ryan, qui n'aurait vu dans le libéralisme et le catholicisme aucune incompatibilité foncière. La tradition religieuse et la modernité politique n'étaient nullement irréconciliables à ses yeux, pour peu que deux conditions fussent respectées. D’une part, la hiérarchie ecclésiastique devait accepter que le laïcat jouât un rôle plus étendu à l'intérieur comme à l'extérieur de l'Église : le clergé devait cesser de se considérer comme le seul intermédiaire entre Dieu et les hommes, de même qu'il devait reconnaitre que l'Étatprovidence pouvait mieux s'acquitter que lui des responsabilités sociales qui lui avaient incombé historiquement. À l'heure de l'aggiornamento, tous les espoirs semblaient permis de ce côté : l'Église paraissait effectivement renouer avec la tradition évangélique et tolérer une certaine démocratisation de son fonctionnement.

D'autre part, l'État avait le devoir d'aborder la question religieuse dans un esprit semblable de modération. Plutôt que de combattre le catholicisme et d'y éliminer toute référence sous prétexte de moderniser la société, il était préférable que l'État adoptât une attitude de neutralité religieuse et permît à toutes les croyances de coexister paisiblement et sans discrimination sur son territoire. Cette neutralité religieuse, cependant, ne devait pas tenir lieu d'obstacle à la reconnaissance des droits scolaires des deux grandes religions qui avaient exercé une influence profonde sur l'évolution historique du Québec, soit le catholicisme et le protestantisme. Voilà pourquoi Ryan revendiquait le maintien du caractère 
confessionnel du système scolaire québécois, malgré la priorité qu'il accordait au libéralisme dans son édifice conceptuel. Comme en matière de langue d'enseignement, il accepta qu'on fit une entorse au principe de neutralité religieuse afin de respecter certaines réalités historiques. Mais pour le reste, l'auteur soutient que le catholicisme de Ryan demeurait en tout temps subordonné à son libéralisme.

Un libéral nationaliste, un libéral catholique, mais lorsque acculé au pied du mur, un libéral d'abord et avant tout : voilà, en un mot, le portrait que brosse Olivier Marcil de Claude Ryan. Le soin que le directeur du Devoir mit à réconcilier et à mettre en équilibre des idéologies souvent antagonistes relevait parfois du tour de force. Le lecteur est en droit de s'interroger sur la réussite de pareille entreprise intellectuelle - et plus particulièrement sur la place véritable qu'occupait le nationalisme dans l'esprit de Ryan - mais il s'avérera dorénavant difficile de commettre l'erreur grossière d'imputer son esprit de modération à la mollesse de ses convictions. Le credo politique de Ryan, on le voit bien, n'était pas moins solide que celui des fédéralistes "purs » et des souverainistes qu'il combattait avec le même acharnement, à un point tel qu'il semble que sa cible préférée ait été par-dessus tout le dogmatisme idéologique, de quelque part qu'il soit venu. Le sempiternel avocat du diable qu'il était développa une pensée complexe et, disons-le franchement, compliquée, une pensée qui défie les interprétations faciles et dont les innombrables nuances le poussèrent à frôler la contradiction, voire le paradoxe à plus d'une reprise.

Bien menée, cette étude aurait toutefois gagné à évoquer plus clairement le contexte intellectuel et historique dans lequel baigna Claude Ryan avant son entrée au Devoir. Cela aurait permis de répondre à un certain nombre de questions qui demeurent en suspens. Quelle est la part dans sa pensée de l'héritage des intellectuels nationalistes qui l'ont précédé ? 
Où se situait-il par rapport aux bouleversements idéologiques que le Canada français avait connus au lendemain de la Deuxième Guerre mondiale? Dans quelle mesure la profonde mutation économique, sociale et culturelle qu'avait subie le Québec après 1945 influença-t-elle l'évolution de sa propre idéologie? Si on exclut les références rapides et peu nombreuses que fait l'auteur à l'École de Montréal et à Lionel Groulx, le lecteur, sur cette question, reste un peu sur sa faim. Ces imprécisions en engendrent d'autres, notamment lorsque l'auteur affirme que le mouvement "néonationaliste » était issu de la Révolution tranquille et qu'il avait abandonné le critère ethnoculturel pour ne plus retenir que les critères politique et territorial dans la définition de la nouvelle "nation" québécoise (pp. 89, 266). Comme le démontrent les travaux de Jean Lamarre (Le Devenir de la nation québécoise) et Michael Behiels (Prelude to Quebec's Quiet Revolution), le mouvement néonationaliste prit forme dès les années 1940, au lendemain de la Deuxième Guerre mondiale. Ce qui le distinguait du nationalisme "traditionnel » n'était pas son abandon de la dimension ethnoculturelle de la nation canadienne-française (cela ne viendrait que bien plus tard), mais plutôt sa volonté d'en comprendre l'évolution historique à l'extérieur du cadre normatif de la morale chrétienne.

Sur le plan des sources et de la méthode, cette étude exploite abondamment les publications de Claude Ryan, notamment les innombrables éditoriaux qu'il a publiés dans $L e$ Devoir de 1962 à 1978. Il aurait été intéressant, s’il est vrai que l'intellectuel évolue dans un réseau de relations et de connaissances, de repérer les influences contemporaines de Ryan. Existe-t-il des sources manuscrites (sa correspondance personnelle, par exemple) que l'historien peut facilement consulter? Un court portrait biographique de Ryan, en le situant plus clairement dans son contexte historique, n'aurait pas été 
dénué d'intérêt. La genèse d'une idéologie, jamais spontanée, importe dans son analyse et sa compréhension.

Il semble que l'impact de Claude Ryan dans la vie publique québécoise et canadienne ait été moins grand durant les années 1960 et 1970 - dominées comme elles le furent par les titans Trudeau et Lévesque - que plus tard, au cours de la décennie suivante. L'idée du statut particulier, en effet, serait au cœur du débat constitutionnel lorsque Brian Mulroney et Robert Bourassa feraient leur entrée à Ottawa et à Québec, d'autant plus que Ryan compterait parmi les ministres les plus influents du cabinet Bourassa. Dans un même ordre d'idées, où se trouve, de nos jours, la progéniture intellectuelle de Claude Ryan? On se souvient du débat que l'«affaire Michaud» a provoqué, il y a quelque temps, autour de la définition de la «nation québécoise ». L'interprétation "ryanienne » d'une nation politique unie par le seul lien de la citoyenneté a-t-elle connu, ironiquement, des lendemains plus heureux au Parti québécois qu'au Parti libéral du Québec? Faut-il voir en Claude Ryan le précurseur d'intellectuels comme Gérard Bouchard?

Ces interrogations ne menacent pas la valeur de l'interprétation globale de l'auteur, qui réussit à démontrer que Claude Ryan mérite de se retrouver dans le panthéon des intellectuels les plus influents de la deuxième moitié du $\mathrm{XX}^{\mathrm{e}}$ siècle. Bien structuré et écrit dans une langue correcte et accessible (en dépit d'un certain nombre de coquilles ici et là), cet ouvrage est d'une lecture agréable et plaira aussi bien aux amateurs d'histoire intellectuelle et politique qu'aux spécialistes en la matière.

Michel Bock

Centre de recherche en civilisation canadienne-française Université d'Ottawa 